\title{
The Metal Penetrating Trauma of Soft Palate in an Adult
}

\author{
Saime Sağıroğlu', Aykut Urfalığlu², Aydemir Koçarslan³, Selman Sarıca' \\ 'Department of Otorhinolaryngology Kahramanmaraş Sütcü Imam University School of Medicine, Kahramanmaraş, Turkey \\ 2Department of Anesthesia, Kahramanmaraş Sütcü Imam University School of Medicine, Kahramanmaraş, Turkey \\ 'Department of Cardiovascular Surgery, Kahramanmaraş Sütcü Imam University School of Medicine, Kahramanmaraş, Turkey
}

Cite this article as: Sağıroğlu S, Urfalıoğlu A, Koçarslan A, Sarıca S. The Metal Penetrating Trauma of Soft Palate in an Adult. J Emerg Med Case Rep 2018; 9(4): 65-7.

\begin{abstract}
Introduction: Impalement injuries of the soft palate and oropharynx are common. The treatment protocols in such cases vary according to the patient and foreign body. A thorough medical history, followed by careful clinical and radiographic examinations, is necessary in management of penetrating injuries.

Case Report: A 20-year-old male patient was admitted to our clinic due to a penetrating injury after a fall onto an iron wire. One end of the iron wire was hanging out of his mouth. The other end of the iron wire had traumatized the oropharynx at the level of the lateral pillar soft palate. Computed tomography angiography showed the wire adjacent to the internal carotid artery (ICA). FB was extracted through oral approaches under general anesthesia.

Conclusion: We report a case of a penetrating FB that injured the soft palate and extended laterally into the ICA. In addition, in this report, the importance of a thorough investigation for FBs is also emphasized.

Keywords: Soft palate, penetrating injury, adult, internal carotid artery

Received: 29.12.2017 Accepted: 07.02.2018
\end{abstract}

\section{Introduction}

Impalement injuries of the soft palate and oropharynx are common in children. A penetrating foreign body (FB) in the neck is of particular concern because it is very close to vital structures. The overall mortality in such cases ranges from $0 \%$ to $11 \%$ (1). The treatment protocols vary according to the patient and FB. The surgeon and the anesthetist must work very carefully and be well familiarized with the treatment protocol during removal of an implanted FB because these injuries, although often not having permanent sequelae, have the potential to cause destructive neurological complications. Thus, a careful assessment should be made in the early hours after the trauma and the patient should be followed closely during treatment.

Here we report an unusual case of a metal FB (an iron wire) penetrating the oral cavity of an adult. The purpose of this report was to evaluate and analyze the management of patients with retained FBs so as to prevent the complications encountered.

\section{Case Report}

\section{Preoperative course}

A 20-year-old male was admitted to the Otorhinolaryngology (ENT) Department with an oral penetrating FB. An iron wire was seen protruding through his mouth (Figure 1). The piece of iron wire extending outside from the mouth showed movement 
with pulsation. No excessive bleeding, vomiting, loss of consciousness, respiratory distress, subcutaneous emphysema, bruit, thrill, stridor, or neurological deficit was observed. The pulsation of the

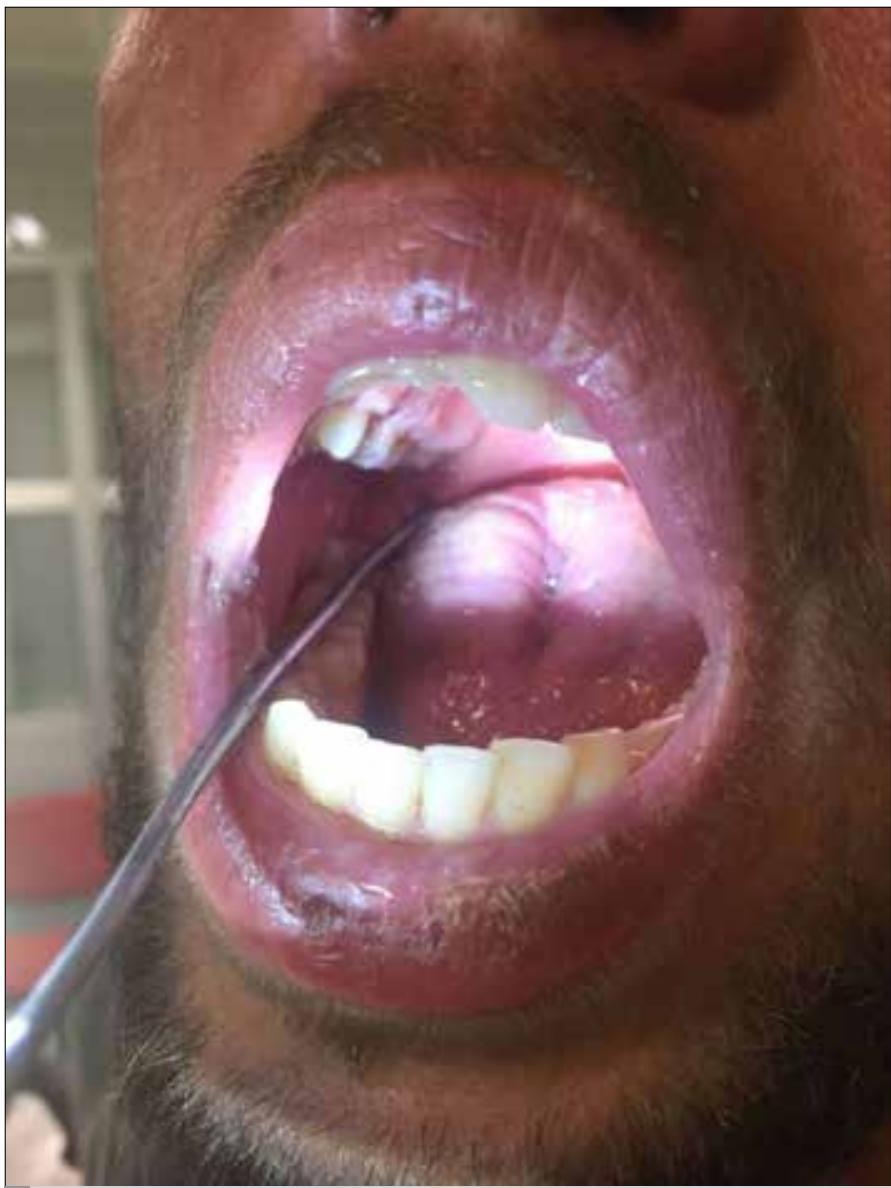

FIGURE 1. Impalement of iron wire.

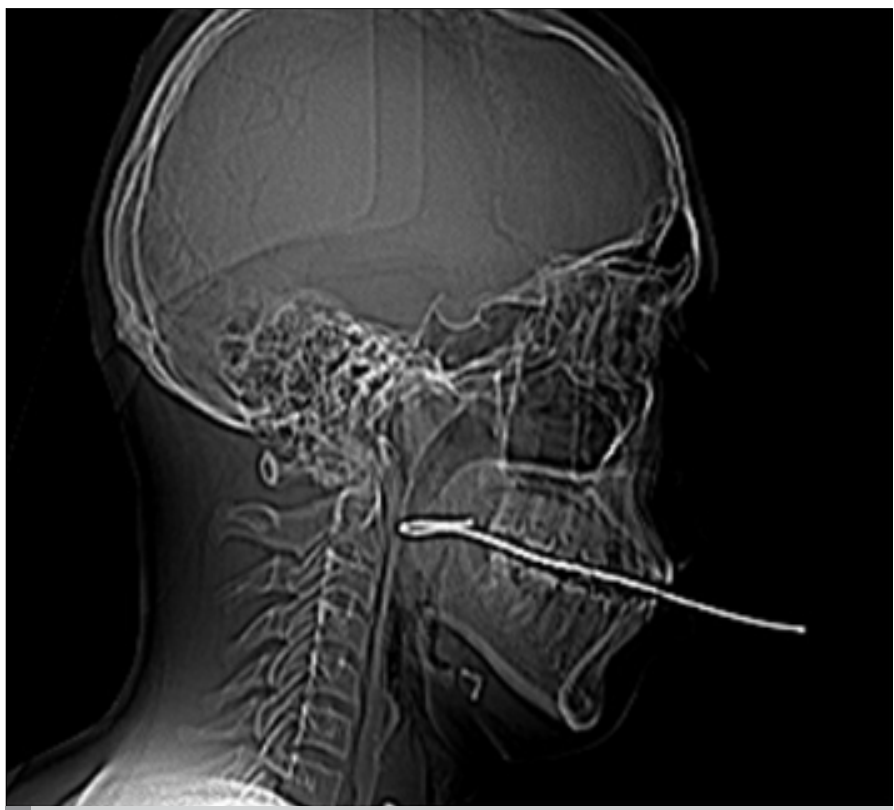

FIGURE 2. X-ray image. carotid and superficial temporal arteries was normal bilaterally. The remaining ENT examination, routine laboratory results, and systemic examination did not show any abnormality.

\section{Imaging methods}

$X$-ray revealed FB (Figure 2). The surgical team assessed the patient and decided that a computed tomography angiography (CTA) of the neck, with contrast, was necessary for further delineation of the vascular structures prior to attempting a surgical manipulation (Figure 3). The CTA evaluation led to the interpretation of FB as a posteriorly curved, metallic, linear FB extending to the right retromolar area along the soft palate. Common air densities extended posteriorly from FB to the adjacent styloid bone. Air densities were also observed at the temporomandibular joint level. FB extended through the mouth toward the neck and also showed proximity to the internal carotid artery (ICA). No vascular injury or hematoma formation was detected.

\section{Operative course}

Vascular surgery and anesthesia consultations were obtained, and a decision was made for exploratory surgery. Two units each of erythrocyte suspensions and fresh frozen plasma were administered be-

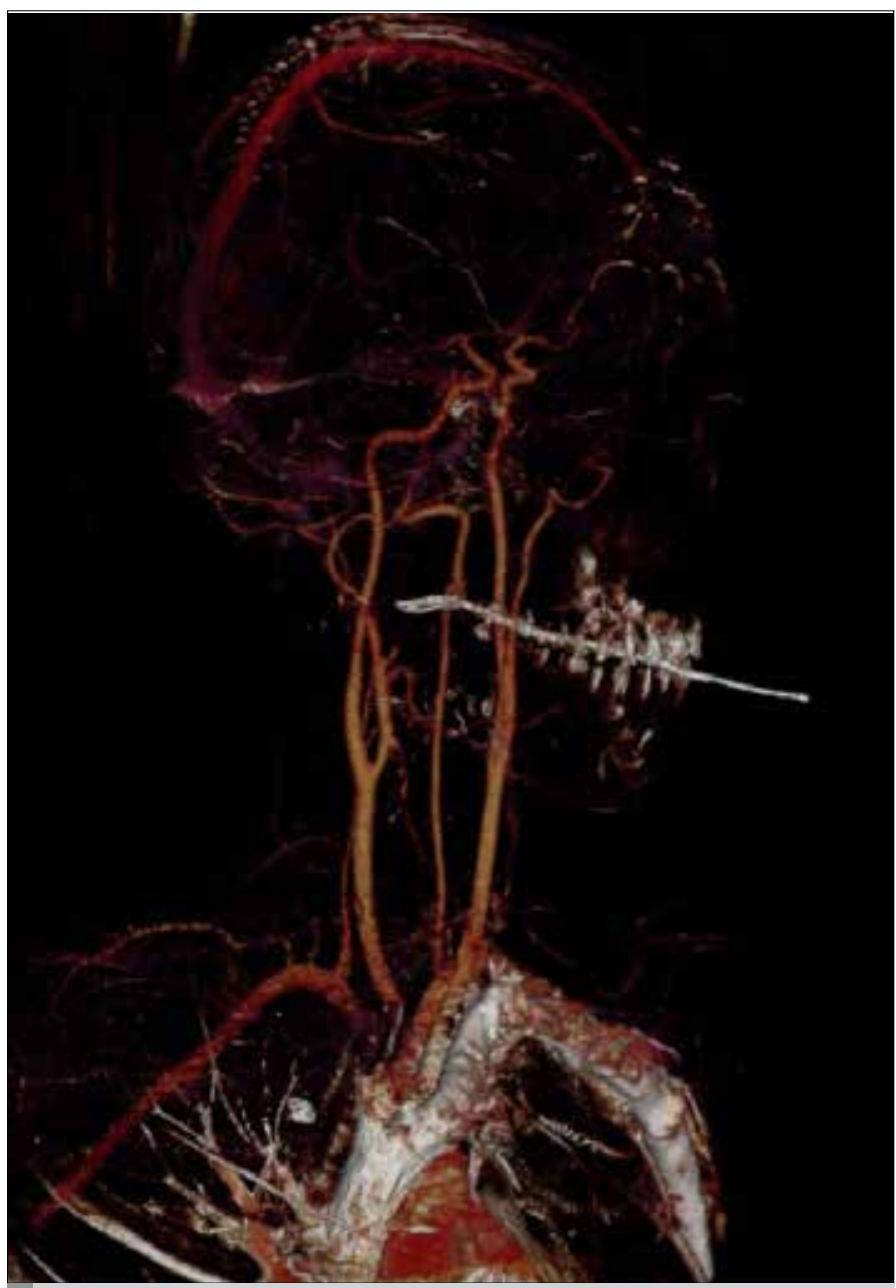

FIGURE 3. CTA scan showing adjacent vessels with the foreign body. 
cause of the risk for intraoperative bleeding following FB removal. FB, which appeared to be near ICA, was carefully cut so that it would remain in the mouth but allow for proper mask ventilation. During the mask ventilation, care was taken not to hyperextend the neck due to the possibility that FB could cause damage to ICA. The oropharyngeal wound was explored by making an oropharyngeal incision along FB. FB extended laterally into the deep palate musculature. Using an anteroposterior approach, an approximately $5 \mathrm{~cm}$ incision was made superior to the right tonsil cavity using the iron wire as a guide. The incision was deepened parallel to the iron wire until the wire was released from the soft tissue. The wire had twisted during impalement and had become hooked to the soft tissue. ICA could be clearly seen, and the adventitia was found to be intact. FB did not damage any major structures and major blood vessels around it and was excised without any problems. Bleeding was controlled by covering the site with a Surgicel ${ }^{\circledR}$. We sutured the deep muscle layer and the oral mucosa, and the surgery was completed without any complications. Intravenous antibiotics, analgesics, and tetanus prophylaxis were administered in appropriate doses.

\section{Discussion}

Impalement injuries of the soft palate and oropharynx occur very rarely in adults. The need for surgical closure of palatal injuries reportedly varies from $7 \%$ to $72 \%(2,3)$. Surgical exploration was performed in only $18 \%$ cases of FB impalement and was reserved for large avulsion flaps or FB (2). Most cases have been observed to heal without any intervention. For example, Takenoshita et al. (4) reported that most soft and hard palate lacerations heal very well; notably, the authors suggested that sutures may actually cause further damage.

Penetrating trauma due to a FB may result in a major vascular injury. In cases like the present one, the patient and/or persons accompanying the patient should not make any attempt to remove the iron wire because an adverse move made during the removal of metal FBs can threaten the patient's life. The patient may go into shock due to excessive bleeding or may die. In such cases, shock should be managed and the airway should be secured by tracheostomy.

X-ray studies of the neck, chest, and abdomen are routinely conducted to localize FBs. However, non-metallic FBs may not be visible in traumatic regions; metal FBs are much easier to visualize using imaging methods. FB may not be detected in a computed tomography $(\mathrm{CT})$ scan, but the $\mathrm{CT}$ scan shows the integrity of the organs, as well as the course, tract, and location of FB in the neck (5). Magnetic resonance angiography, Doppler ultrasonography, and other radiological investigations can provide important information about vital structure injury. Angiography or frequent Doppler studies are recommended in cases with a strong suspicion of ICA injury.

Potentially serious sequelae can frequently occur following an injury of this nature. These include deep neck abscess, widespread emphysema, internal carotid thrombosis, and stroke (6-9). Lateral soft palate injury with subsequent ICA occlusion and neurologic deficit may also occur, and destructive neurological complications, such as ce- rebral ischemia caused by thrombosis of ICA, can arise (9). Given the delayed onset of neurological signs, this life-threatening situation can be easily overlooked (10). As a result, various treatment protocols have been recommended for the management of impalement injuries, such as hospitalization for up to $48-72 \mathrm{~h}$ of observation. If necessary, such patients should be treated with anticoagulants.

\section{Conclusion}

In conclusion, a thorough medical history, followed by careful clinical and radiographic examinations, is a necessity for penetrating injuries to the palate. The treatment objectives include uneventful healing of the injured tissues, as well as restoration of normal function. In our opinion, the use of standard surgical approaches to extract a FB reduces the complications encountered peri- and postoperatively.

Informed Consent: Written informed consent was obtained from patients who participated in this study.

Peer-review: Externally peer-reviewed.

Author Contributions: Concept - S.S.; Design - A.U.; Supervision - A.K., S.S.; Resources - A.U., S.S.; Materials - A.K., A.U.; Data Collection and/or Processing - A.K., S.S.; Analysis and/or Interpretation - S.S., S.S.; Literature Search - S.S.; Writing Manuscript - S.S., S.S.; Critical Review - S.S., A.K.

Conflict of Interest: The authors have no conflict of interest to declare.

Financial Disclosure: The authors declared that this study has received no financial support.

\section{References}

1. Nason RW, Assuras GN, Gray PR, Lipschitz J, Burns CM. Penetrating neck injuries: Analysis of experiences from a Canadian trauma centre. Can J Surg 2001; 44: 122-6.

2. Hellman JR, Shott SR, Gootee MJ. Impalement injuries of the soft palate in children: review of 131 cases. Int J Pediatr Otorhinolaryngol 1993; 26: 157-63. [CrossRef]

3. von Domarus $\mathrm{H}$, Poeschel W. Impalement injuries of the palate. Plast Reconstr Surg 1983; 72: 656-8. [CrossRef]

4. Takenoshita Y, Sasaki M, Horinouchi Y, Ikebe T, Kawano Y. Impalement injuries of the oral cavity in children. ASDC J Dent Child 1996; 63: 181-4

5. Gracias VH, Reilly PM, Philpott J, Klein WP, Lee SY, Singer M, et al. Computed tomography in the evaluation of penetrating neck trauma:a preliminary study. Arch Surg 2001; 136: 1231-5. [CrossRef]

6. Law RC, Fouque CA, Waddell A, Cusick E. Lesson of the week. Penetrating intra-oral trauma in children. BMJ 1997; 314: 50-1. [CrossRef]

7. Rowley H, Christian J, Dennis A. Pharyngeal perforation: an easily missed finding following intra-oral injury. J Accid Emerg Med 1995; 12: 145-6. [CrossRef]

8. Hengerer AS, De Groot TR, Rivers RJ, Pettee DS. Internal carotid artery thrombosis following soft palate injuries: a case report and review of 16 cases. Laryngoscope 1984; 94: 1571-5. [CrossRef]

9. Graham CJ, Schwartz JE, Stacy T. Stroke following oral trauma in children. Ann Emerg Med 1991; 20: 1029-31. [CrossRef]

10. Suskind DL, Tavill MA, Keller JL, Austin MB. Management of the carotid artery following penetrating injuries of the soft palate. Int J Pediatr Otorhinolaryngol 1997; 39: 41-9. [CrossRef] 